\title{
ITCM: Improved Adaptive Transmission Control Mechanism for Underwater Sensor Network
}

\author{
Yiming $\mathrm{Yan}^{1}$, Xiaodong Liu ${ }^{2}$ a \\ ${ }^{1}$ College of Information Science and Engineering, Ocean University of China, \\ Qingdao, 266100, China \\ ${ }^{2}$ College of Mathematical Science, Ocean University of China, \\ Qingdao, 266100, China \\ aemail: liuxiaodong.ouc@hotmail.com
}

Keywords: Underwater acoustic sensor networks; Bit error rate; Transmission Control Mechanism

\begin{abstract}
An Improved Adaptive Transmission Control Mechanism (IATC) for underwater acoustic sensor networks is proposed in this paper. This mechanism controls transmission delay and releases collisions adaptively according to different conditions of the wireless links periodically feed back by the receiver. Our work is succeeding in releasing collisions and reducing propagation delay. Further more, the energy is reserved by reducing repeated transmission. Simulations show IATC can amend the transmission time adaptively depending on users and can save $50 \%$ energy.
\end{abstract}

\section{Introduction}

The past decades many researchers have highlighted the importance of monitoring aquatic environments for scientific, environmental and commercial reasons. There has existed much work focusing on highly precise, real-time, fine grained spatiotemporal sampling of the ocean environment, current methods such as remote telemetry and sequential local sensing cannot satisfy many application needs, which call for wireless underwater networking. Underwater Wireless Sensor Networks (UWSNs) enable applications for oceanographic data collection, pollution monitoring, offshore exploration, tsunami warning, assisted navigation and mine reconnaissance [1]. Underwater nodes exchange signals mainly in acoustic channels. The optical wave was an alternative option in UWSNs. However, optical waves are affected by scattering in the underwater environment. Moreover, the transmission of optical signals requires high precision in pointing the narrow laser beams. Thus, links in underwater networks are based on acoustic communications technology. This situation proposes us new challenges due to the harsh acoustic environment in Underwater Acoustic Sensor Networks (UASNs), such as:

- The propagation delay in underwater is five orders of magnitude higher than in radio frequency (RF) terrestrial channels [1]. This is a critical issue especially in surveillance or in environmental monitoring applications such as seismic monitoring. Therefore how can we control transmission in an efficient and time sensitive manner is a crucial issue.

- Energy consumption of transmission is much higher in UASNs. Sensors' batteries are hardly recharged in ocean. Thus the energy resumption is more important.

One of the best ways to address the aforementioned problems is to minimize collisions. We have proposed a transmission control mechanism for UASNs in 2008 [2]. In this paper we propose another Improved Adaptive Transmission Control Mechanism (IATC) to control the transmission time.

\section{Related Work}

Underwater network is a rather unexplored area although underwater communications have been experimented since World War II. In 1945, underwater telephone was developed in the United States to communicate with submarines. Yang et al. developed sensor networks for application to liquid environments in 2002[3]. The efficient transmission is extensively investigated in many 
literatures. There are many algorithms which are efficient in Wireless Sensor Networks (WSNs). Tian et al. developed a novel component that fits seamlessly into the ground based sensor network communication stack [5]. The algorithm AIDA successfully reduced end-to-end delay and transmission energy consumption in WSNs. Then as an extension, a bit error rate guided packet length optimization algorithm (BGPP) is proposed by Zeng peng et al. [6]. They used a lossless data aggregation method to adjust the packet length and successfully reduced retransmission by recursion in WSNs. From discussions in this paper we know that this algorithm is more efficient when BER is lower. But these two algorithms are not suitable for channels with long intermittences and high error rates such as acoustic channels. Khan et al. also presented an adaptive self configuring in-network storage model for data persistency in WSNs. All this works released collisions efficiently in ground based sensor networks but are not suitable for UASNs.

We proposed an Adaptive Transmission Control (ATC) mechanism for UASNs in [2]. This mechanism can dampen congestion and reduce the number of collisions dynamically according to the change of children nodes, BER of acoustic channels and the limitation of propagation delay. But ATC can not amend the propagation delay adaptively depending on the floating of nodes. To amend this, in this paper we propose an improved mechanism (IATC), where users can change the limitation of propagation adaptively. This mechanism can dampen congestion and reduce the number of collisions dynamically according to the topology of the network, BER and the limitation of propagation delay. These lead to fewer retransmissions and can save energy efficiently.

\section{Design of IATC}

In this section, we firstly illustrate the topology of IATC. As shown in Fig. 1, the network has a tree-structure and all nodes floating in a restricted area. After that we describe three important steps of IATC in detail. To any node ( $\mathrm{v}$, for example), layer $(v)$ is denoted as hops between sink and $v$, layer $(\operatorname{sink})=0$. Let $v_{i j}$ denotes node of the tree, where $i$ stand for the layer, and $j$ for sequence number of the same layer. Let $V=\left\{v_{i j}\right\}$, the structure is shown in Fig. 1.

Let $S\left(v_{i_{0} j_{0}}\right)=\left\{v_{i j} \mid v_{i j} \in V, i=i_{0}+1, j \in J_{i_{0} j_{0}}\right\}$ denote the aggregation of all child nodes of $v_{i_{0} j_{0}}$ in the RFS network, where $J_{i_{0} j_{0}}$ contains all the sequence number of these nodes. Because of the movement of nodes, $S\left(v_{i_{0} j_{0}}\right)$ and $J_{i_{0} j_{0}}$ are variables. For each node according to $B E R$, the definition of the node's Weight for transmission time $(\mathrm{w})$ is given as follows:

$$
w\left(v_{i j}\right)=\frac{1}{(1-B E R)^{(H+D)}}
$$

It is easy to know that $w\left(v_{i j}\right)$ is the average number of packets required for successfully transferring $\mathrm{D}$ bits data. Let $\mathrm{n}$ denote the number of packets received from a same children node; let $n_{w}$ denote the number of bad packets of $n$. Then we give the definition of the Final Weight as follows:

Definition 1: to any node $v_{i_{0} j_{0}}$ in the RFS network, the Final Weight (FW) of node $v_{i_{0} j_{0}}$ is specified by the maximum of FW of it's child nodes, that is:

$$
f w\left(v_{i_{0} j_{0}}\right)=t w\left(v_{i_{0} j_{0}}\right)+w\left(v_{i_{0} j_{0}}\right)
$$

Where $t w\left(v_{i_{0} j_{0}}\right)=\max _{j \in J_{i_{0} j_{0}}}\left\{|\bar{B}-B|^{\left(N-n_{w}\right)} \times f w\left(v_{i_{0}+1, j}\right)\right\}$

Where $N=n \times B, \quad \bar{B}=n_{w} / n$ that is: $t w\left(v_{i_{0} j_{0}}\right)=\max _{j \in J_{i_{0} j_{0}}}\left\{\left[\left|\frac{n_{w}-B}{n}\right|\right]^{\left(n \times B-n_{w}\right)} \times f w\left(v_{i_{0}+1, j}\right)\right\}$

It is straight forward the Final Weight of leaf nodes is: $f w\left(v_{\text {depth }, j}\right)=w\left(v_{\text {depth, }}\right)$

Let all nodes send packets in a fix frequency and assume there was a given transmission time 
before the transmission time is confirmed. This mechanism is mainly built up by the following two parts: the first one is to get the FW of all nodes; the next step is work out the transmission time of each node according to the WF and the limitation of propagation delay.

\section{A. Get the FW for Each Node}

In the algorithm IATC, each intermediate node $v_{i j}$ should get and storage WF of their own. So that a REQUEST_FW message should be carried in packets sent from the sink to intermediate nodes, as a response all nodes of the tree (except the sink) should reply the REPLY_FW message, which contains their FW, to their parent node. It worth noting that there is no need to send REQUEST_FW and REPLY_FW separately; they all could be carried by other packets.

In this process, leaf nodes on the Depth layer reply REPLY_FW messages to their parent nodes firstly, then nodes on the Depth-1 layer will execute the algorithm 1 shown as follows:

According to WF carried by REPLY_FW messages, each intermediate node works out it's own FW which is stored and sent to the parent node in a new REPLY_FW message. Then their parent nodes will carry on until the sink finally receives all the REPLY_FW messages from nodes on the first layer and then it will store the FW of each root node as the Final Weight of this child tree. For example, in Figure 1 the child tree $\left\{v_{11}, v_{21}, v_{22}, v_{31}, v_{41}, v_{42}, v_{43}\right\}$ denoted by $L_{1}$, so fw $\left(L_{1}\right) f w\left(v_{1 j}\right)$. And through this paper we let $f w\left(v_{1 j}\right)$ stand for the Final Weight of this child tree $L_{j}$.

\section{B. Get the Transmission Time of Each Node}

In this paper we assume that all sensors are sensing the environment at a fixed rate. Transmission time of each node will be worked out according to the limitation of propagation delay and the WF. After users gave the limitation of propagation delay (denoted by $T$, the sink would firstly work out the upper limit of propagation delay of child tree $L_{j}: \Delta \mathrm{T}\left(\mathrm{L}_{\mathrm{j}}\right)=\mathrm{T}-\mathrm{fw}\left(\mathrm{v}_{1 \mathrm{j}}\right) \mathrm{p}^{-1}$, Where $p$ stands for the fixed $f$ transferring rate of nodes. It is clearly that while the network topology was given, $\Delta T\left(L_{j}\right)$ mostly depend on the limitation of propagation delay $T$. After getting the upper limit of propagation delay for each child tree, a GET-TIME message carrying the information of $T, \Delta T\left(L_{j}\right)$ and $f w\left(v_{1 j}\right)$ will be passed to every child tree.

To nodes on different layers, the following expression is used to work their own transmission time:

$$
\Delta T\left(v_{i k}\right)=\frac{f w\left(v_{i k}\right)}{f w\left(v_{1 j}\right)} \times \Delta T\left(L_{j}\right)+\frac{f w\left(v_{i k}\right)}{p}
$$

It is easy to know $\Delta T\left(L_{j}\right)$ is changing according to $T$.

From the discussion we know (3) is suitable for all these conditions. When a node get the GET-TIME, according to WF which has been stored by itself, algorithm 2 will be performed to work out the transmission time of the node.

\section{Simulation Result and Discussion}

In our simulation, all nodes will obey the SPT(Shortest Paths Tree) scheme to construct the tree-structure. Thus we get a tree-structure in our simulation as in Figure 1(a). The sink node is settled on $(600,0)$.

With limited power resources, it is vital for sensor nodes to minimize energy consumption during radio communication to extend the lifetime of the sensor network. So we will evaluate the performance gain achieved by IATC. Here we compare IATC with other transmission time control mechanisms and discuss their effect on energy saving in these five schemes.

The following two transmission time control mechanisms will be compared: 1) IATC 2)ATC ${ }^{[2]}$

In this paper we just consider consumption of energy. As shown in Figure 1(b) we know IATC consumes less energy than ATC algorithm in these five schemes. This means that the retransmission 
caused by channel contention is minimized by IATC. Especially when the BER is higher than $0.5 \times 10^{-3}$, the energy retrenched by IATC is much lower. The energy consumption of IATC is about $40.91 \%$ of ATC. From these we know that IATC is more efficient in dampening congestion and reducing the number of collisions under worse conditions.

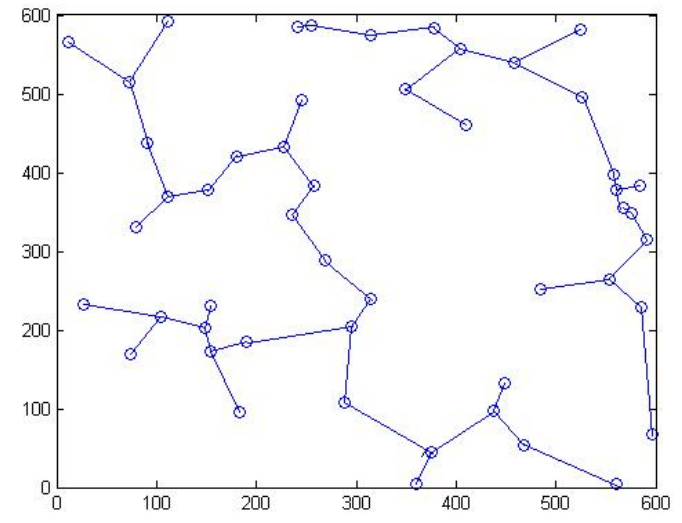

(a) Topology of Simulation

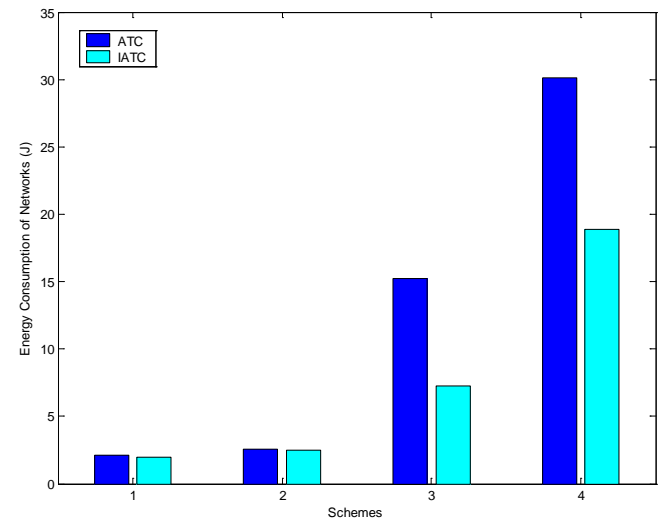

(b) Energy Consumption

Fig.1. The experimental results

\section{Conclusion}

In this paper we introduce IATC, An adaptive transmission control mechanism for underwater acoustic sensor networks. Due to the highly dynamic and unpredictable nature of acoustic communication in underwater sensor networks, a novel feedback-based scheduling scheme is proposed to dynamically adapt to reducing energy consumption and response time. In our experiments we evaluate the performance gain achieved by IATC. As shown in our evaluation, IATC can reduce energy consumption efficiently without sacrificing end-to-end delay and this would be more efficient while the traffic condition is worse. So our work would be more useful in the worse wireless transmission conditions of underwater networks. Moreover, significantly IATC adjust the response time under requirements.

\section{References}

[1] Raghunathan V.,Schurgers C.,Park S.,et. Energy Aware Wireless Microsensor Networks [J]. IEEE Signal Processing Magazine,2002, pp.40-50.

[2] Z.W. Guo, X.D. Liu, Y. Feng, An Adaptive Transmission Control Mechanism for Underwater Acoustic Sensor Network. Proc of IEEE NPC’08[C]. Shanghai, 2008.

[3] Heinzelman W., Chandrakasan A., Balakrishnan H., Energy-Efficient Communication Protocol for Wireless Microsensor Networks[A], the $33^{\text {th }}$ Annual Hawaii International Conference on System Sciences [C]. Hawaii, 2003, pp.01-10.

[4] He Tian, Blum Brian M, Stankovic John A.,Abdelzaher T. AIDA: Adaptive Application Independent Aggregation in Sensor Networks [J].ACM Trans on Embedded Computing System, 2003, pp. 426-457.

[5] ZENG peng,YU hai-bin,LIANG wei,Energy Effici-ency Packet Optimization Algorithm for Wireless Sensor Network[J].Control and Decision, 2006, pp.180-183.

[6] Lettieri P.,Srivastava M B.,Adaptive Frame Length Control for Improving Wireless Link Throughput, Range and Energy Efficiency [A]. Proc of IEEE INFOCOM'98[C].San Francisco, 1998, pp.564-571. 